ARTICULO DE INVESTIGACION

\title{
Composición química y densidad básica relativa de la madera de dos especies arbustivas de encino blanco de la Sierra de Álvarez, SLP, México
}

\author{
Chemical composition and relative basic density of two \\ shrub white oak wood species from the Sierra de Álvarez, \\ SLP, México
}

\author{
Guadalupe M. Bárcenas-Pazos¹, Rosalva Ríos-Villa2, \\ J. Rogelio Aguirre-Rivera ${ }^{3}$, Bertha I. Juárez-Flores ${ }^{3}$ \\ y J. Amador Honorato-Salazar4
}

\begin{abstract}
RESUMEN
En la sierra de Álvarez, San Luis Potosí, se han identificado 18 especies de encinos; de ellas, Quercus sebifera y Q. tinkhami son de hábito arbustivo y pertenecen a la sección Quercus o encinos blancos. A la fecha se desconocen estudios sobre las características de su madera, la cual sólo se aprovecha localmente en forma reducida y rudimentaria. En este trabajo se presenta su caracterización química. Se evaluaron los contenidos relativos de lignina, extractos y cenizas, así como la densidad básica relativa y el contenido de celulosa. Se calcularon los estadísticos básicos para cada componente y se realizó un análisis de varianza entre especies para cada parámetro estimado. La cantidad de celulosa y lignina de ambas especies fue estadísticamente similar ( $p>95 \%$ ); además, los valores obtenidos coinciden con los presentados por otros autores para encinos mexicanos. La cantidad de cenizas fue mayor que lo publicado para especies mexicanas del mismo género, lo cual posiblemente se deba a las condiciones de crecimiento de estas especies, particularmente el clima y el tipo de suelo. Sólo los contenidos de extractos de $Q$. sebifera con la mezcla etanol-benceno y con agua caliente fueron mayores que los registrados para otros encinos blancos. El contenido de extractos fue estadísticamente mayor en Q. sebifera. La riqueza de cenizas y extractos de estas especies parece estar relacionada con su adaptación a las condiciones de aridez en las que crecen. La densidad básica relativa de $Q$. sebifera es media y la de $Q$. thinkami, es alta.
\end{abstract}

PALABRAS CLAVE:

Composición química, extractos, madera de encino, Quercus, San Luis Potosí.

Programa Multidisciplinario de Posgrado en Ciencias Ambientales, Universidad Autónoma de San Luis Potosí. Altair 200. Fracc. del Llano. 78377 San Luis Potosí, SLP. Unidad de Recursos Forestales, Instituto de Ecología, A.C., Xalapa, Ver. Correo electrónico: guadalupe.barcenas@inecol.edu.mx. Posgrado en Ciencias Biomédicas, Universidad Autónoma de San Luis Potosí. Instituto de Investigación de Zonas Desérticas, Universidad Autónoma de San Luis Potosí. Altair 200. Fracc. del Llano. 78377 San Luis Potosí, SLP.

$4 \quad$ INIFAP Campo Experimental San Martinito, Centro de Investigación Regional Centro. Km. 56.5 Carretera Federal México-Puebla, San Martinito. Tlahuapan, Municipio Tlahuapan, Puebla. CP 74100. 


\section{ABSTRACT}

In the mountain range of Álvarez, San Luis Potosí eighteen oak species have been identified; two of them (Quercus sebifera and Q. tinkhami) included into the Quercus section or white oaks are shrubs. Currently, studies are not known on the wood characteristics of these species, which are only used locally in a reduced and rudimentary way. Their chemical composition is presented here. Essays to estimate relative content (\%) of lignin, extracts and ashes were carried out; specific gravity was also calculated along with volume of cellulose. Basic statistics were estimated for each parameter obtained. Variance analyses were applied between the essays sets of cellulose, lignin, ash, and extracts. Not significant differences ( $p>95 \%$ ) were found between species for cellulose and lignin. These results were similar to those presented by other authors for Mexican oaks. Ash volume resulted to be greater; it may be due to their particular climate and soil type, mainly. The extracts of $Q$. sebifera obtained by the ethanol-benzene mixture and by hot water were greater than those published for white oaks. These species were different $(p>95 \%)$ for extract content. Ash and extracts richness of these species seems to be related to their aridity adaptations. Relative basic density values for $Q$. sebifera were medium and high for Q. tinkhami.

\section{KEYWORDS:}

Chemical composition, extracts, oakwood, Quercus, San Luis Potosí.

\section{INTRODUCCIÓN}

En México, en nivel de género, los encinos son considerados como el segundo recurso forestal maderable más importante después del género Pinus (Rzedowski, 2006 y Nixon, 1993). La madera de encino posee un valor muy alto como materia prima cuando es procesada adecuadamente; sin embargo, debido a su dureza, su distribución geográfica menos homogénea que la de los pinos y la variabilidad específica en sus características tecnológicas, su uso más común aún es como combustible (García, 1995 y Zavala, 1990).

Un argumento utilizado reiterativamente para justificar el desuso de la madera de encino en productos con mayor nivel de proceso, es el desconocimiento de sus cualidades y reacciones, además de su variabilidad en dependencia de la zona geográfica donde es cortada (De la Paz, 2000). En México se cuenta con información, particularmente sobre su ecología y distribución, sobre varias especies de encinos (Carter, 1953; Martínez, 1981; Nixon, 1993 y Valencia, 2004).

Sobre las características tecnológicas de la madera de encinos, De la Paz (2000) estableció la relación entre las características anatómicas y las propiedades físicas y mecánicas de la madera de 24 especies de encinos, 16 de ellos rojos y ocho blancos. Quintanar (2002) publicó una recopilación de diversos trabajos sobre taxonomía, distribución, aprovechamiento y usos actuales, características anatómicas, propiedades físicomecánicas, composición química, índices de rendimiento y calidad de fibra para pulpa, durabilidad, aserrío, secado y maquinado de la madera de 31 especies de encinos rojos y 16 de encinos blancos.

La composición química de la madera de los encinos mexicanos se ha estudiado sólo para unas pocas especies y en aspectos generales y específicos, que van desde el pH hasta la caracterización de algunos compuestos químicos de la madera y de la corteza, según lo resumido por Honorato (2002). Así, Delgado (1980) estimó el contenido de cenizas, extractos con agua caliente y con etanolbenceno, así como $\alpha$-celulosa y hemicelulosa en la madera de dos especies de encino rojos ( $Q$. laurina y $Q$. candicans) y dos blancos ( $Q$. resinosa y $Q$. obtusata) del estado de Jalisco; el porcentaje de 
lignina lo calculó por diferencia. Fuentes (1980) determinó el contenido de carbohidratos estructurales, extractos con etanolbenceno y lignina de la madera del fuste y de las ramas de Quercus resinosa, otro encino blanco también del estado de Jalisco.

En la madera de dos especies de encinos rojos ( $Q$. laurina y $Q$. candicans) provenientes del estado de Michoacán, Rutiaga et al. (2000 a y b) estimaron los porcentajes de lignina, carbohidratos estructurales y cenizas, y cuantificaron el porcentaje de extractos con tres disolventes, éter de petróleo, acetona y metanol. Por su parte, Bárcenas (2002) analizó el efecto de la lignina y los extractos removidos con disolventes orgánicos sobre dos propiedades físicas de la madera de varias especies; una de ellas fue el encino rojo $Q$. laurina.

Bautista y Honorato (2005) estudiaron la composición química de la madera de $Q$. coccolobifolia, $Q$. durifolia y $Q$. rugosa recolectados en el estado de Guanajuato, y de $Q$. oleoides proveniente del centro del estado de Veracruz. Los dos primeros son encinos rojos y las otras dos especies son encinos blancos. En todos los casos, menos para lignina, los encinos blancos presentaron valores mayores de los componentes estudiados.

En general, los valores de alfa celulosa publicados para la madera de encinos mexicanos van de $37 \%$ a $56 \%$, de hemicelulosas de $22 \%$ a $30 \%$ y de lignina de $8 \%$ a $22 \%$. Para las holocelulosas (celulosa y hemicelulosas) se han encontrado valores de $60 \%$ a $82 \%$; específicamente para hemicelulosas, formadas básicamente por pentosas, se han registrado contenidos de $18 \%$ a $23 \%$ (Honorato, 2002). La cantidad de celulosa presente en la madera de los encinos mexicanos estudiados, es mayor que la registrada en las especies de otros países, mientras que en los otros compuestos son similares (Honorato, 2002).

Con respecto a los componentes menores, la cantidad relativa de cenizas en la madera de los encinos mexicanos oscila entre $0,32 \%$ y $1,38 \%$; la de sílice varía de $0,0025 \%$ a $0,0093 \%$; la de extractos con agua caliente varía de $4,61 \%$ a $10,00 \%$, con etanol-benceno de $1,14 \%$ a $7,21 \%$ y con hidróxido de sodio al $1 \%$ de $20,89 \%$ a $26,00 \%$; y el contenido de taninos varía desde $0,59 \%$ hasta $33,40 \%$ (Honorato, 2002).

Las especies de encino que crecen en la sierra de Álvarez de SLP, han sido identificadas y estudiadas, ecológicamente (García, 1995; García et al.; 1999, García y Aguirre a y b, inéditos; Ramírez, 2000; Castillo, 2003 y Castillo et al., 2008), pero sobre las características de su madera se desconocen antecedentes, particularmente sobre las dos especies arbustivas consideradas en este trabajo.

\section{OBJETIVO}

Cuantificar los componentes químicos y determinar la densidad básica relativa de la madera de dos especies arbustivas de encinos blancos ( $Q$. tinkhami C.H. Müll. y Q. sebifera Trel.) de la sierra de Álvarez, SLP.

\section{MATERIALES Y METODOS}

\section{Recolección de material}

Las muestras de las especies estudiadas se recolectaron en la sierra de Álvarez, área natural declarada para protección forestal y refugio de fauna silvestre (Figura 1). Este sistema orográfico está localizado al sureste de la capital del estado San Luis Potosí, y se extiende en dirección NO-SE, con altitudes de 


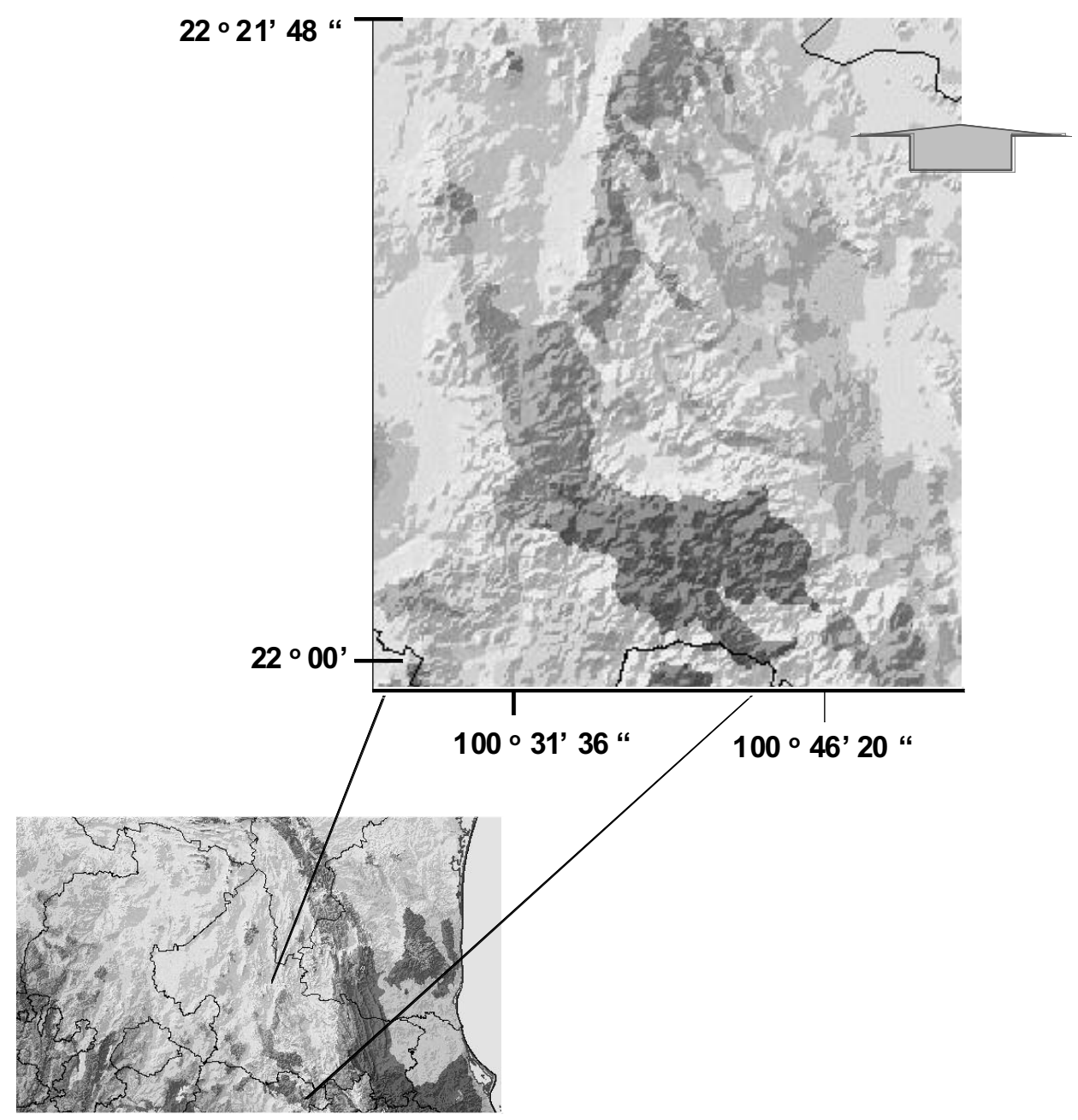

Figura 1. Ubicación de la sierra de Álvarez, SLP

2000 a 2700 msnm. Este sistema y las serranías que le suceden hacia el norte forman un límite natural entre la cuenca del valle de San Luis Potosí y la cuenca de Rioverde (García et al., 1999). En la sierra de Álvarez predomina un clima semiseco templado con lluvias en verano, del tipo $\mathrm{BS}_{1} \mathrm{kw}$ (García, 1988), con precipitación media anual de $366 \mathrm{~mm}$ a 571 $\mathrm{mm}$, un porcentaje de lluvia invernal entre $5,0 \%$ y $10,2 \%$, y la cantidad de lluvia del mes más húmedo de la mitad caliente del año es por lo menos diez veces mayor que la del mes más seco. La temperatura media anual es de $17^{\circ} \mathrm{C}$ a $18^{\circ} \mathrm{C}$, y sus variaciones diarias son de $-3^{\circ} \mathrm{C}$ a $18^{\circ} \mathrm{C}$ en el mes más frío (García et al., 1999). Se registran dos temporadas al año, la seca de noviembre a abril y la lluviosa, de mayo a octubre; los meses de precipitación abundante coinciden con los meses de temperaturas más elevadas (Ramírez, 
2000). La sierra está formada predominantemente por rocas sedimentarias (calizas y lutitas), pero en la porción sur hay manchones de rocas ígneas. Predominan los suelos de tipo litosol éutrico (García et al., 1999).

Se recolectaron tallos de dos individuos de Q. tinkhami en la comunidad de San José de Magaña, y de tres individuos de $Q$. sebifera, a un lado del camino hacia la comunidad de El Pato, ambos pertenecientes al municipio de Armadillo de los Infante en la sierra de Álvarez, SLP. Las dos especies recolectadas pertenecen a la sección Quercus (Valencia, 2004) o encinos blancos. De cada especie se recolectó material botánico como ejemplares botánicos de respaldo, los cuales se procesaron y depositaron en el
Herbario Isidro Palacios (SPLM) del Instituto de Investigación de Zonas Desérticas de la Universidad Autónoma de San Luis Potosí.

La descripción general de las especies recolectadas en la sierra de Álvarez es la siguiente (García y Aguirre a, inédito):

Quercus tinkhami C.H. Müll. (Figura 2) Nombre común encino chaparro. Arbustos caducifolios de aproximadamente $3,0 \mathrm{~m}$ de alto, con tallos hasta de $8,0 \mathrm{~cm}$ de diámetro, de rectos a torcidos. Su hábitat, en la sierra de Álvarez, SLP es el bosque de galería y el bosque subhúmedo de encino, sobre sustratos de origen sedimentario e ígneo.

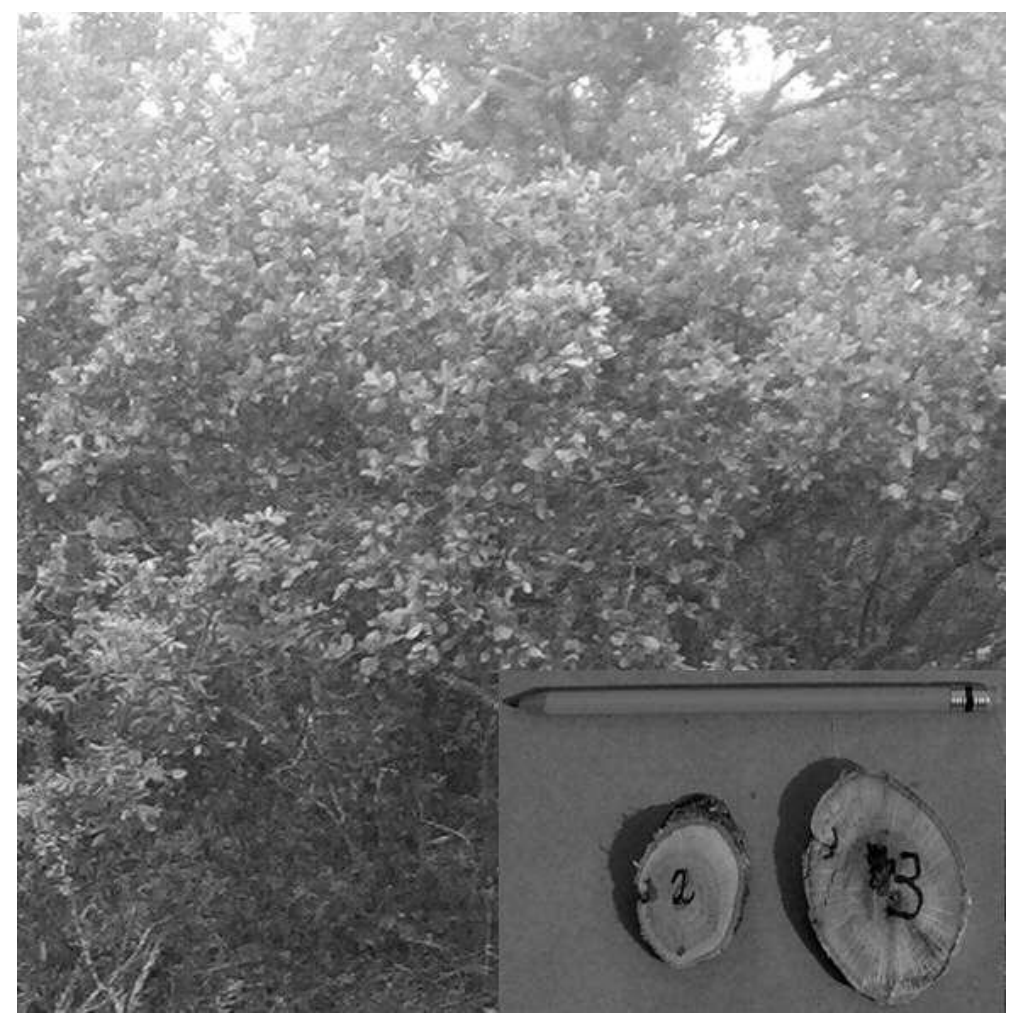

Figura 2. Quercus tinkhami C. H. Müll. 


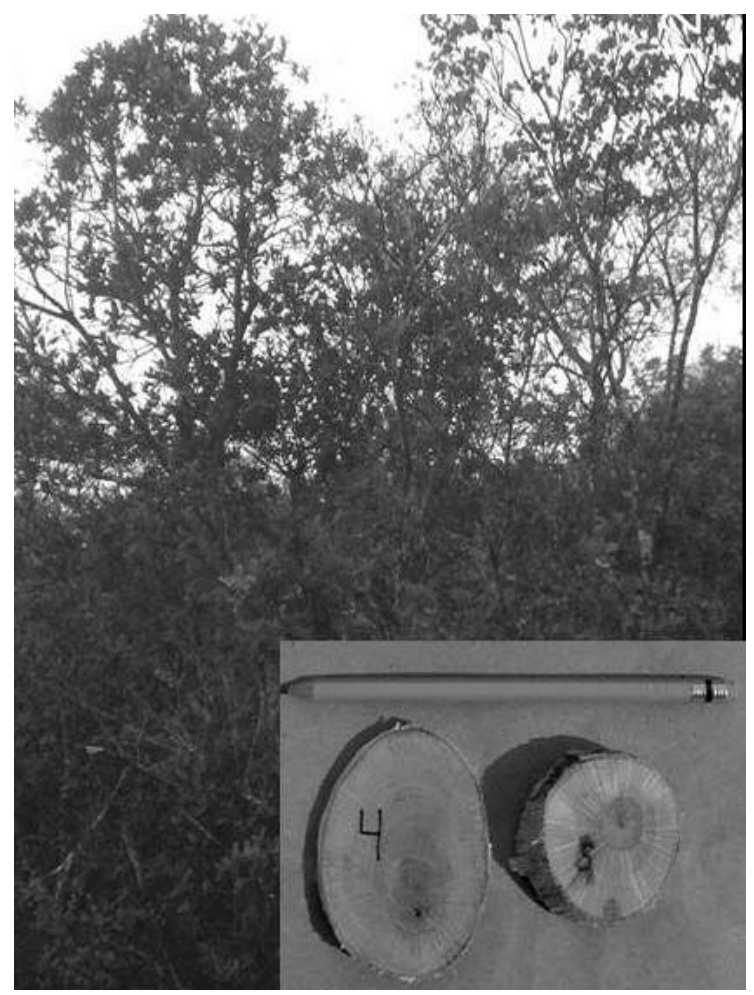

Figura 2. Quercus sebifera Trel.

Q. sebifera Trel. (Figura 3) Nombre común, encino. Arbustos caducifolios de aproximadamente $3,0 \mathrm{~m}$ de altura, tallos con diámetros hasta de $15,0 \mathrm{~cm}$, rectos a ligeramente torcidos. En la sierra de Álvarez, SLP, su hábitat es el bosque de encino y crece en sustratos de origen sedimentario.

\section{Preparación del material}

Los tallos recolectados se identificaron con un número consecutivo; luego se descortezaron y se procesaron para volverlos astillas antes de que se redujera considerablemente el contenido de humedad de su madera. Posteriormente, las muestras hechas astillas se secaron al aire libre, hasta que su contenido de humedad se equilibró con el ambiente. Enseguida se pulverizaron en un molino Wiley para homogeneizarlas, se tamizaron a través de una malla número 40 y se recolectó lo que quedó retenido en un tamiz con malla número 60 . El material seco y tamizado se mantuvo en bolsas selladas para minimizar los cambios en su contenido de humedad, hasta ser analizadas.

\section{Análisis químicos}

Para la determinación de los contenidos de celulosa y lignina se removieron las sustancias solubles (extractos) no estructurales mediante una extracción secuencial con disolventes con un gradiente creciente de polaridad, de acuerdo con lo establecido en la norma Preparation of extractive free-wood, TAPPI Test Method T $257 \mathrm{~cm}-85$ (TAPPI, 1998). La muestra extraída se dejó secar 
al aire libre hasta que su humedad se estabilizara con la humedad ambiental; entonces se guardó en un recipiente sellado, para minimizar sus cambios de humedad, hasta que se realizaran las determinaciones de celulosa y lignina.

El contenido de humedad $(\mathrm{CH})$ de las muestras se estimó antes de cada ensayo, de acuerdo con lo establecido en la norma Preparation of extractive free-wood, TAPPI Test Method T $257 \mathrm{~cm}-85$ (TAPPI, 1998). El método utilizado para determinar el contenido relativo de celulosa fue el desarrollado por Kuschener y Hoffer en 1929 (Browning, 1967). El contenido de lignina se realizó siguiendo lo establecido en la norma Acidinsoluble lignin in wood and pulp, TAPPI Test Method T 222 om-88 (TAPPI, 1998). El contenido de cenizas se determinó de acuerdo con lo que establece la norma Ash in wood, TAPPI Test Method T211 om-85 (TAPPI, 1998). Para la determinación de los extractos con agua caliente y con etanolbenceno se usaron las normas Water solubility of wood and pulp, TAPPI Test Method T 207 om-88 y Solvent extractives of wood and pulp, TAPPI Test Method T 204 om-88 (TAPPI 1998), respectivamente.

Para ambas especies se determinó la densidad básica relativa (DR), de acuerdo con lo establecido por la norma ASTM D-2395-94, Método B. De cada especie se seleccionaron cinco especímenes y se registró su volumen verde, esto es madera recién derribada (VV). Posteriormente, se secaron al aire bajo sombra y luego en horno eléctrico a $105^{\circ} \mathrm{C} \pm 3^{\circ} \mathrm{C}$, hasta que alcanzaron peso constante en dos pesadas consecutivas, con lo cual se obtuvo su peso anhidro (PA). Los valores de densidad básica están relacionados con la densidad del agua, por lo que carecen de unidades.

Todas las estimaciones químicas se hicieron en dos ensayos independientes con cuatro repeticiones. Para establecer la signi- ficación de las diferencias entre las dos especies se aplicó una prueba de $\mathrm{F}$ (análisis de varianza, $p \geq 95$ ) a los resultados obtenidos para cada atributo evaluado.

\section{RESULTADOS}

Los resultados de los ensayos químicos para los compuestos estructurales y cenizas se presentan en la Tabla 1. Los resultados obtenidos para los extractos se presentan en la Tabla 2, así como los valores de densidad básica relativa.

En cuanto a que es celulosa, el compuesto estructural más abundante de la madera, el contenido relativo en $Q$. tinkhami $(43,37 \%)$ fue estadísticamente similar al de Q. sebifera $(45,72 \%)$ (Tabla 1). Menores diferencias aun se encontraron en el contenido de lignina (Q. tinkhami, 21,02\% y $Q$. sebifera, 21,47\%). En cambio, el contenido de cenizas de $Q$. tinkhami $(2,67 \%)$ fue estadísticamente menor que el de $Q$. sebifera (3,22\%) (Tabla 1).

Con respecto a los extractos, los que se obtuvieron con agua caliente fueron más abundantes que los separados con la mezcla de disolventes orgánicos (Tabla 2). A la vez, en ambos casos se registraron diferencias significativas entre especies. Así, los porcentajes de extractos con agua caliente en la madera de $Q$. tinkhami $(7,67)$ y en la de $Q$. sebifera $(9,16)$ superaron significativamente a los de $Q$. tinkhami $(4,50)$ y $Q$. sebifera $(6,13)$ obtenidos con la mezcla de disolventes. A la vez, el contenido relativo de extracto acuoso en la madera de $Q$. sebifera $(9,16 \%)$ fue estadísticamente mayor que el de $Q$. tinkhami $(7,67 \%)$, y el contenido de extractos con disolventes de $Q$. sebifera $(6,13 \%)$ también superó significativamente al de Q. tinkhami $(4,50 \%)$ (Tabla 2). La densidad relativa (pa/vv) de $Q$. thinkamii $(0,735)$ fue significativamente mayor que la de $Q$. sebifera $(0,678)$. 


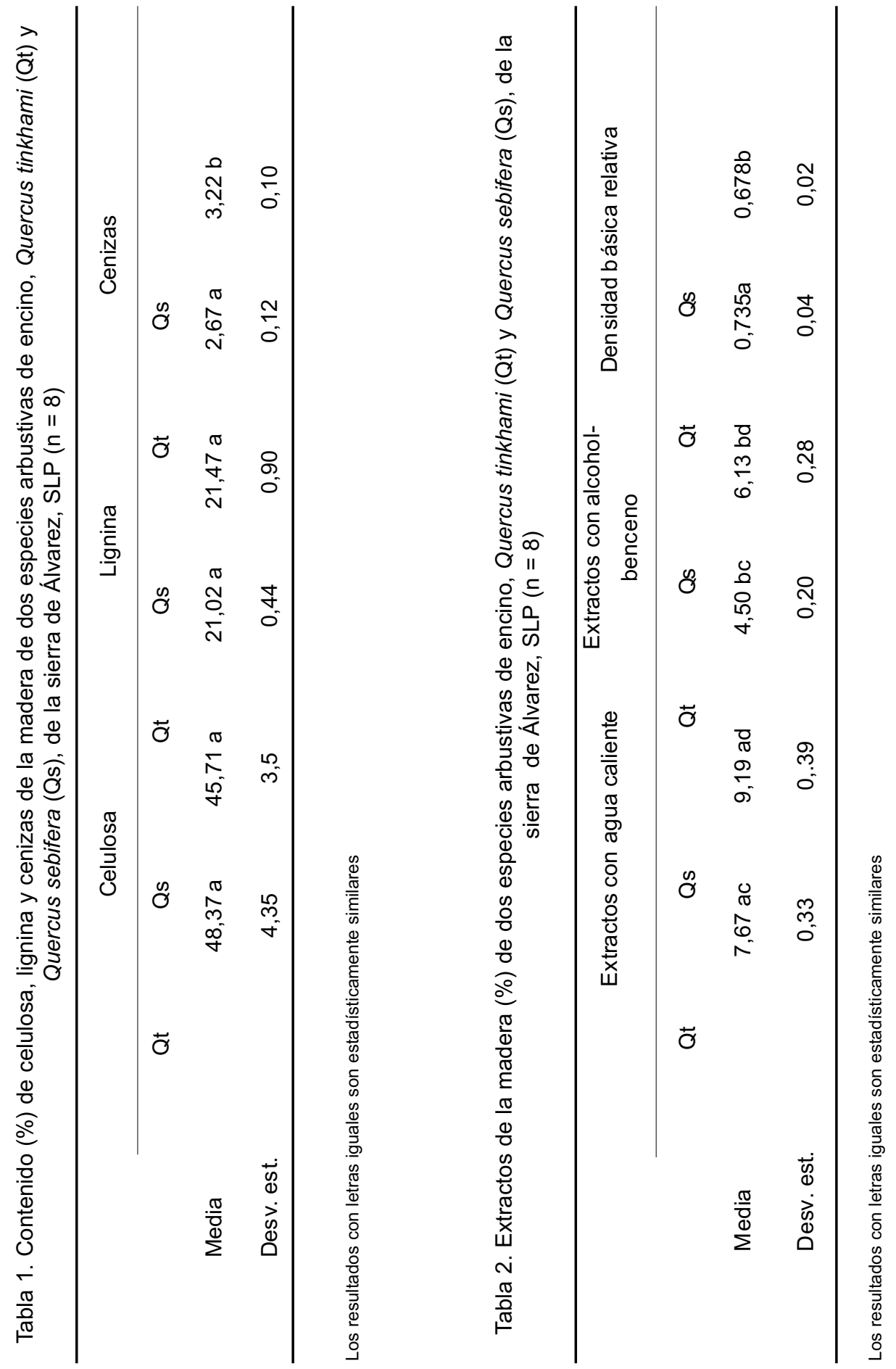




\section{DISCUSIÓN}

Los porcentajes de celulosa y lignina encontrados en la madera de los encinos arbustivos de sierra de Álvarez (Tabla 1), son equiparables con los registrados en general para la madera de encinos mexicanos (Honorato, 2002). Específicamente para los encinos blancos mexicanos Honorato (2002) resume que el intervalo del contenido relativo de celulosa es de $51,94 \%$ a $56,43 \%$ y que el de lignina es de $19,84 \%$ a $22,57 \%$. Los contenidos de celulosa obtenidos son también comparables a los obtenidos por Bautista y Honorato (2005) para dos encinos blancos $(Q$. rugosa del estado de Guanajuato y $Q$. oleoides del estado de Veracruz) $52,94 \%$ y $51,68 \%$, respectivamente. Los contenidos relativos de lignina registrados por estos autores son $20,40 \%$ y $22,37 \%$, respectivamente. Así, ambas especies estudiadas presentaron contenidos de celulosa y lignina dentro del intervalo obtenido para otros encinos blancos del país. Para algunos encinos blancos de Estados Unidos, usados en tonelería, entre ellos Q. alba, Rowell et al. (2005) presentan porcentajes de celulosa entre $40 \%$ y $47 \%$ y de lignina de $24 \%$ a $28 \%$, de manera que los valores de celulosa obtenidos de la madera de $Q$. sebifera son similares y los de la madera de $Q$. tinkhami son más altos. En cambio, los valores de lignina de los encinos arbustivos de sierra de Álvarez son menores que los presentados por Rowell et al. (2005). Bodirlau et al. (2007) en un estudio de la composición química de varias especies maderables, establecieron que el contenido relativo de celulosa y lignina de $Q$. robur, encino blanco europeo usado en tonelería, varía de $42,79 \%$ a $45,02 \%$ y de $23,32 \%$ a $24,82 \%$, respectivamente; así el contenido de celulosa es menor en esta especie que en Q. sebifera y $Q$. tinkhami, pero el porcentaje de lignina es mayor en las dos especies mexicanas.
Los contenidos de cenizas registrados en las especies estudiadas (Tabla 1) son mayores que los valores menores que $1,0 \%$, descritos por Honorato (2002) para la madera de varias especies mexicanas de Quercus, y a los registrados por Bautista y Honorato (2005) para la madera de $Q$. rugosa $(0,45 \%)$ y $Q$. oleoides $(0,74 \%)$. La procedencia de todos los ejemplares cuyos resultados están publicados, es siempre más septentrional que los estudiados en este trabajo. Saka (2001) menciona que en la madera de especies intertropicales, la cantidad de cenizas puede llegar a ser hasta de $5 \%$. Es posible que el contenido de cenizas encontrado se deba a que se trata de encinos meridionales, propios de ambientes subhúmedos a secos. De acuerdo con García y Aguirre (Inédito b), el hábitat de $Q$. sebifera es más seco (lomas y laderas abiertas; sustrato sedimentario) que el de Q. tinkhami (bosque de galería y bosques aledaños subhúmedos, sustrato ígneo y sedimentario). Así, el contenido de cenizas estadísticamente mayor de $Q$. sebifera puede representar una adaptación fisiológica a condiciones de potencial hídrico del suelo más restrictivas que las prevalecientes en el hábitat de Q. tinkhami. Bautista y Honorato (2005), en contraste, encontraron que para $Q$. oleoides, de clima cálido y húmedo, es más alto el contenido de cenizas, pero siempre menor que $1,0 \%$; sin embargo, esta especie está vinculada a suelos muy restrictivos sobre afloramientos ígneos (Pennington y Sarukhán, 2005). En comparación con los contenidos de cenizas que presentan Rowell et al. (2005) para algunos encinos blancos de Estados Unidos $(0,3 \%$ a $1,2 \%)$, los calculados en este estudio son mayores. Esto concuerda con lo señalado por Saka (2001) de que las especies maderables intertropicales presentan mayores contenidos de sustancias inorgánicas que las boreales. En efecto, Bodirlau et al. (2007) encontraron en $Q$. robur, porcentajes de 
cenizas entre $0,14 \%$ y $1,30 \%$, valores menores que los registrados para $Q$. tinkhami y $Q$. sebifera.

Los extractos de la madera son sustancias sin una función estructural completamente conocida; pueden ser grasas, grasas ácidas, alcoholes grasos, fenoles, terpenos, esteroides, resinas ácidas, ceras y otros componentes orgánicos menores (Rowell et al., 2005). En la mayoría de las especies, el porcentaje de los extractos en el peso seco de la madera, es menor que 10\%; sin embargo, en algunas puede llegar hasta $30 \%$ (Reyes et al., 1987 y FPL, 1999).

Los extractos removibles con disolventes orgánicos incluyen resinas, ácidos grasos y sus ésteres, ceras, sustancias no saponificables, colorantes, hidrocarburos no volátiles, carbohidratos de bajo peso molecular y algunas sales (Saka, 2001). El contenido relativo de extractos con los disolventes orgánicos en la madera de Q. tinkhamii, $(4,50 \%)$ es cercano al punto medio del intervalo $(2,79 \%$ a $5,24 \%)$ recopilado por Honorato (2002), y del encontrado por Bautista y Honorato (2005) $(3,05 \%$ y $4,82 \%)$ para dos encinos blancos, $Q$. rugosa y $Q$. oleoides, respectivamente. En cambio, el porcentaje registrado para $Q$. sebifera $(6,13 \%)$ supera claramente el límite mayor de dicha amplitud. La diferencia significativa entre la madera de ambas especies (Tabla 2), puede estar igualmente relacionada con la diferencia correspondiente en el contenido de cenizas, esto es, que el mayor contenido de extractos en la madera de $Q$. sebifera contribuye probablemente a generarle una mayor capacidad osmótica y por lo tanto una mayor tolerancia a la aridez (Baeza y Freer, 2001; Braun-Blanquet, 1979 y Weiler, 2004), y que la mayor riqueza de cenizas se relacione también con la posibilidad de la reutilización de los nutrientes de la albura cuando hay escasa disponibilidad de los mismos en el suelo (Penninckx et al., 2001).

Entre los extractos que son removidos con agua se encuentran carbohidratos solubles, algunos ácidos inorgánicos, materiales fenólicos, y algunas sustancias inorgánicas (Baeza y Freer, 2001). El agua lava algunos compuestos también removibles con disolventes orgánicos (Rowell et al., 2005), lo que sumado a la mayor presencia de sustancias como polisacáridos o flavonoides, los cuales son muy solubles en agua (Browning, 1967), puede explicar la diferencia entre los obtenidos con agua y con los disolventes orgánicos en las maderas de este estudio.

El porcentaje de las sustancias extraídas con agua caliente en la madera de $Q$. tinkhami $(7,67)$ está cerca del límite superior del intervalo para otros encinos blancos mexicanos que resume Honorato (2002) $(4,99$ a 7,76), y del que registraron Bautista y Honorato (2005) para Q. rugosa y $Q$. oleoides $(4,97$ y 7,97$)$. Asimismo, dicho porcentaje se encuentran dentro del intervalo presentado por Rowell et al. (2005) para los encinos blancos estadounidenses $(6,0$ a 9,0), y por Bodirlau et al. (2007) para Q. robur $(7,56$ a 8,12). En cambio, el porcentaje registrado para $Q$. sebifera $(9,19)$ supera claramente todos los valores consignados. Al igual que los extractos con agua caliente, el mayor contenido relativo de los extractos con disolventes orgánicos puede ser una respuesta de adaptación a suelos áridos (Baeza y Freer, 2001; Braun-Blanquet, 1979 y Weiler, 2004).

La densidad relativa de las dos especies $(0,735$ de $Q$. tinkhami y 0,678 de $Q$. sebifera) está dentro de los valores registrados para otros encinos blancos en el país 0,688 a 0,818 (Bárcenas y Dávalos, 2001); está dentro de los valores de los encinos estadounidenses $(0,66$ a 0,72$)$ 
presentada en FPL (1999) pero es mayor que la de $Q$. robur $(0,50$ a 0,66$)$, encino blanco europeo (Bodirlaw et al., 2007). De acuerdo con la clasificación de Dávalos y Bárcenas (1999) para maderas mexicanas la densidad de $Q$. tinkhami es muy alta (> $0,700)$ y la $Q$. sebifera es alta $(0,550$ a 0,690). La densidad más alta de $Q$. tinkhami se puede atribuir a su mayor densidad de fibras que de vasos o a sus paredes celulares más gruesas (Panshin y DeZeew, 1980) pues sus porcentajes de compuestos estructurales y extractos fueron menores que los de $Q$. sebifera.

Este trabajo constituye una aportación pionera significativa sobre el conocimiento de las características la madera de estas dos especies arburstivas en particular y de las especies de encino de la sierra de Álvarez de San Luis Potosí, que por las condiciones de aridez en las que crecen contrastan con las características de la madera de otras especies del mismo género nativas de climas más húmedos.

\section{CONCLUSIONES}

Los contenidos relativos de celulosa, lignina y cenizas en la madera de $Q$. tinkhami fueron $48,37 \%, 21,02 \%$ y $2,67 \%$, respectivamente. Los porcentajes de extractos fueron $7,67 \%$ para los removidos con agua caliente y $4,50 \%$ con disolventes orgánicos.

Para Q. sebifera los porcentajes obtenidos fueron 45,71 de celulosa, 21,47 de lignina, 3,22 de cenizas, 9,19 de extractos con agua caliente, y 6,13 con la mezcla de etanol-benceno.

Los contenidos relativos de celulosa y lignina de $Q$. thinkami y $Q$. sebifera fueron estadísticamente similares.

Q. sebifera superó estadísicamente a $Q$. tinkhami en contenido relativo de cenizas y de extractos removidos con agua caliente y con disolventes orgánicos.

Los contenidos de celulosa y lignina de Q. tinkhami y $Q$. sebifera son comparables con los publicados para la madera de otras especies de encinos blancos mexicanos y latitudes más septentrionales.

Los contenidos de cenizas y extractos en la madera de $Q$. sebifera son más altos que los registrados para otros encinos blancos mexicanos y de otras latitudes y parecen estar relacionados con su mayor tolerancia a las condiciones de aridez.

La densidad relativa de las dos especies $(0,735$ de $Q$. tinkhami y 0,678 de Q. sebifera) está dentro de los valores registrados para otros encinos blancos en el país; es mayor que la de los encinos estadounidenses y que la de $Q$. robur, encino blanco europeo. La densidad de Q. tinkhami es muy alta y la de $Q$. sebifera es alta.

\section{RECONOCIMIENTOS}

Este trabajo fue realizado con el financiamiento proporcionado por el Fondo de Apoyo a la Investigación (CO5FAl-10.24.45) de la Universidad Autónoma de San Luis Potosí y por el Fondo Sectorial CONAFOR-CONACYT 200641801 al proyecto "Evaluación de la madera de encino blanco para la maduración de bebidas destiladas".

\section{REFERENCIAS}

American Society for Testing Materials. 2002. Standard test methods for specific gravity of wood and woodbased materials D-2395. Annual 
Book of ASTM Standards, Vol 04.10. Philadelphia, USA. 8 p.

Baeza J. y J. Freer. 2001. Chemical characterization of wood and its components. In: Hon, D. N. S. y N. Shiraishi (Eds.). Wood and cellulosic chemistry. 2nd ed. Marcel Dekker. New York. pp. 275-384.

Bárcenas P., G. y R. Dávalos S. 2001. Shrinking values for 106 mexican woods. Journal of Tropical Forest Products 7(2):126-135.

Bárcenas P., G. M. 2002. Efecto del contenido de lignina, extractos, radios y densidad relativa en las contracciones de cinco especies de madera. Tesis de maestría. Colegio de Posgraduados. Montecillos, Edo. de México. México. $61 \mathrm{p}$.

Bautista H., R. y J. A. Honorato S. 2005. Composición química de la madera de cuatro especies del género Quercus. Ciencia Forestal 30(98):25-50.

Bodirlau, R., I. Spiridion y C. A. Teaca. 2007. Chemical investigation of wood tree species en temperate forest in East Northern Romania. BioResources (2):41-57.

Braun-Blanquet, J. 1979. Fitosociología. Bases para el estudio de las comunidades vegetales. Ediciones Blume, Madrid. 820 p.

Browning, B. L. 1967. Methods of wood chemistry. Interscience Publishers. New York. Vol. I. 384 p.

Carter, A. 1953. Los encinos de Baja California. Boletín de la Sociedad Botánica de México 56:39-42.

Castillo L., P. 2003. Encinares de sierra de Álvarez, S.L.P.: caracterización y dinámica. Tesis de Maestría. Universidad Autónoma Agraria Antonio Narro. Buenavista, Saltillo, Coahuila, México. 122 p.

Castillo L., P., J. A. Flores C., J. R. Aguirre R. y R. I. Yeaton H. 2008. Dinámica del encinar de la sierra de Álvarez, San Luis Potosí, México. Madera y Bosques 14(1):21-36.

Dávalos S., R. y G. M. Bárcenas P. 1999. Clasificación de las propiedades mecánicas de las maderas mexicanas en condición seca. Madera y Bosques 5(1):61-69.

De la Paz P. O., C. 2000. Relación estructura propiedades físico-mecánicas de la madera de algunas especies de encinos (Quercus) mexicanas. Tesis de Doctorado en Ciencias. Facultad de Ciencias, UNAM, México, D.F. 266 p.

Delgado F., E. 1980. Estudio analítico de los carbohidratos de cuatro especies de encino. Tesis profesional. Facultad de Ciencias Químicas, Universidad de Guadalajara, Guadalajara, Jalisco, México. 104 p.

Forest Products Laboratory (FPL). 1999. Wood as engineering material. Agricultural Handbook No. 72. Forest Service, US Department of Agriculture. Madison, WI. USA. 387 p.

Fuentes M., J. G. 1980. Estudio analítico de los carbohidratos de la madera de Quercus resinosa. Tesis profesional, Universidad de Guadalajara, Guadalajara, Jalisco, México. 109 p.

García, E. 1988. Modificaciones al sistema Köppen-García (para adaptarlo a las condiciones de la República Mexicana). Larios, México, D.F. 219 p. 
García S., F. 1995. Los encinos del valle de San Luis Potosí. II Seminario Nacional sobre utilización de los encinos. INIFAP/SARH Reporte científico número especial $15 \mathrm{Vol}$. I. UANL. Linares, Nuevo León. México. pp. 895-930.

García S., F., J. R. Aguirre R., J. Villanueva D. y J. García P. 1999. Contribución al conocimiento florístico de la sierra de Álvarez, San Luis Potosí, México. Polibotánica 10:73-103.

García S., F y J. R. Aguirre R. Los árboles de la sierra de Álvarez, SLP. 230 p. Inédito $a$.

García S., F y J. R. Aguirre R. Los arbustos de la sierra de Álvarez, SLP. 1461 p. Inédito b.

Honorato S., J. A. 2002. Química de la madera de encinos. In: Quintanar O., J. (Ed.). Características, propiedades y procesos de transformación de la madera de los encinos de México. Libro técnico No. 2. INIFAP. CIRCE. Campo experimental Sn. Martinito. Tlahuapan, Puebla. México. pp. 86-106.

Martínez, M. 1981. Los encinos de México. Anales del Instituto de Biología. 2a. ed., Comisión Forestal de Michoacán. México. Serie Técnica No. 8. 358 p.

Nixon, K. C. 1993. The genus Quercus in Mexico. In: T.P. Ramamoorthy, T. P.; R. Bye; A. Lot y J. Fa (Eds.). Biological diversity in Mexico: origin and distribution. Oxford University Press. New York. pp. 447-458.

Panshin, A. J. y C. de Zeeuw. 1980. Textbook of wood technology. Structure, identification, properties, and uses of the United States and Canada. McGraw-Hill, New York. 722 p.
Pennington, T y J. Sarukhán. 2005. Árboles tropicales de México. Manual para la identificación de las principales especies. $3 a$ ed. UNAM/Fondo de Cultura Económica. México, D.F. 523 p.

Penninckx, V., S. Glineur, W. Gruber, J. Herbauts y P. Meerts. 2001. Radial variation in wood mineral element concentrations: a comparison of beech and pedunculate oak from Belgian Ardennes. Annals of Forests Science 58:253-260.

Quintanar O., J. (Ed.). 2002. Características, propiedades y procesos de transformación de la madera de los encinos de México. Libro técnico No. 2. INIFAP- CIRCE. Campo experimental Sn. Martinito. Tlahuapan, Puebla. México. 194 p.

Ramírez T., H. M. 2000. Análisis estructural del bosque de encino de la sierra de Álvarez, S.L.P. Tesis profesional, Facultad de Agronomía, Universidad Autónoma de San Luis Potosí. México. 46 p.

Reyes Ch., R., V. Pérez M. y S. del Ángel B. 1987. Influencia de los extractos en la resistencia natural de seis maderas tropicales al hongo de pudrición morena Lenzites trabea. Biótica 12(1):7-20.

Rowell, R. M., R. Pettersen, J. S. Han, J. S. Rowell y M. A. Tshabalala. 2005. Cell wall chemistry. In: Rowell, R. M. (Ed.). Handbook of wood chemistry and wood composites. CRC Press, Boca Raton, Florida. USA. pp. 35-74.

Rutiaga Q., J. G., E. Windeisen y C. Strobel. 2000a. Composición química del duramen de la madera de Quercus candicans Neé. Madera y Bosques 6(2):73-80.

Rutiaga Q., J.G., C. Strobel, E. Windeisen y G. Wegener. 2000b. Composición 
química de la madera de un encino. En: Memorias del III Congreso Mexicano de Tecnología de Productos Forestales. Durango, Dgo. México. pp. 93-94.

Saka, S. 2001. Chemical composition and distribution. In: Hon, D. N. S. y N. Shiraishi (Eds.). Wood and cellulosic chemistry. 2nd ed. Marcel Dekker. New York. pp. 51-81.

Technical Association for the Pulp and Paper Industries. 1998. Solvent extractives in wood and pulp. TAPPI Test Method T 204 om-88. TAPPI Press. Atlanta, Georgia. USA. 3 p.

Technical Association for the Pulp and Paper Industries. 1998. Water solubility of wood and pulp. TAPPI Test Method T 207 om-88. TAPPI Press. Atlanta, Georgia. USA. 2 p.

Technical Association for the Pulp and Paper Industries. 1998. Ash in wood and pulp. TAPPI Test Method T 211 om-85. TAPPI Press. Atlanta, Georgia. USA. 2 p.
Technical Association for the Pulp and Paper Industries. 1998. Acid-insoluble lignin in wood and pulp. TAPPI Test Method T 222 om-88. TAPPI Press. Atlanta, Georgia. USA. 3 p.

Technical Association for the Pulp and Paper Industries. 1998. Preparation of extractive free-wood. TAPPI Test Method T 257 om-88. TAPPI Press. Atlanta, Georgia. USA. 4 p.

Valencia A., S. 2004. Diversidad del género Quercus (Fagaceae) en México. Boletín de la Sociedad Botánica de México 75:33-53.

Weiler, E. W. 2004. Fisiología del metabolismo. In: Sitte, P.; E. W. Weiler; J. W. Kadereit; A. Bresinsky y C. Korner. Strasburger Tratado de Botánica. $35 a$ ed. Ed. Omega. España. pp: 223-359.

Zavala Ch., F. 1990. Los encinos mexicanos: un recurso desaprovechado. Ciencia y Desarrollo 16(95): 43-51. Composición química y densidad básica relativa de la madera de dos especies arbustivas de encino blanco de la Sierra de Álvarez, SLP, México. Madera y Bosques 14(3):81-94. 